\title{
Heterometallic (3d-4f) Coordination Clusters with Unique Topology: Self-Assembly Synthesis, Structural Features, and Magnetic Properties
}

\author{
Muhammad Nadeem Akhtar ${ }^{\dagger *}$, Murad A. AlDamen ${ }^{*}$, Javid Khan ${ }^{\S}$, M. Shahid ${ }^{\perp}$ and \\ Alexander M. Kirillovl,, \\ ${ }^{\dagger}$ Department of Chemistry, Baghdad-ul-Jadeed Campus, The Islamia University of Bahawalpur, \\ Bahawalpur 63100, Pakistan \\ ${ }^{\ddagger}$ Department of Chemistry, School of Science, the University of Jordan, Amman 11942, Jordan. \\ ${ }^{\S}$ School of Chemistry, Sun Yat-Sen University, Guangzhou 510275, P. R. China. \\ $\perp$ Functional Inorganic Materials Lab. (FIML), Department of Chemistry, Aligarh Muslim University, \\ Aligarh 202002, India \\ "Centro de Química Estrutural and Departamento de Engenharia Química, Instituto Superior Técnico, \\ Universidade de Lisboa, Av. Rovisco Pais, 1049-001, Lisbon, Portugal.

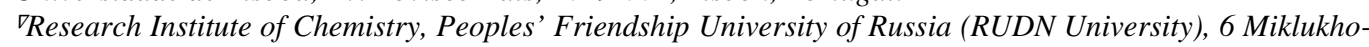 \\ Maklaya st., Moscow, 117198, Russian Federation.
}

Corresponding authors emails: nadeemchem@yahoo.com,m.nadeemakhtar@iub.edu.pk (M. N. Akhtar) maldamen@ju.edu.jo(M.AlDamen)

\section{Table of Contents}

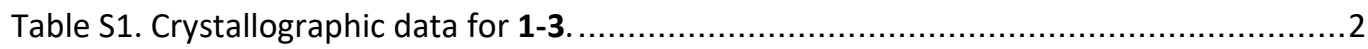

Table S2. Selected distances $(\AA ̊)$ and angles (degree) in the isostructural compounds 1-3......3

Table S3. Shape measurements for $\operatorname{Ln}(\mathrm{III})$ coordination environments in compounds 1-3.....5

Fig. S1. Comparative representation of FTIR spectra of 1-3 (above), important peaks in 1

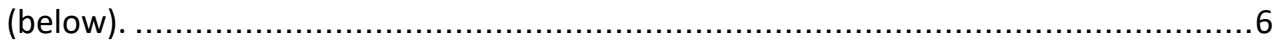

Fig. S2. Experimental and simulated powder X-ray diffraction (PXRD) patterns for 1-3........6

Fig. S3. (Above) Views of 1 along the plane ca. $(-1,0,1)$ and 3 along the plane ca. $(-1,1.5,-1)$, showing a packing pattern and the solvent accessible voids; iron: yellow, nitrogen: blue, oxygen: red, Y/Dy : pink and violet. Below) A molecular unit of 1, showing an intramolecular interaction (green dashed line) between the azide ligand and hydroxyl hydrogen.

Fig S4. Perspective views of $\mathbf{2}$ and $\mathbf{3}$ showing the available internal voids obtained by Olex2. 8

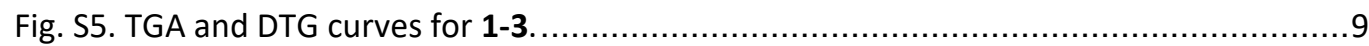

Fig. S6. $\chi_{M}{ }^{-1}$ versus $T$ plots data for $1-3$; inset shows the trendlines equations.................... 10

Fig. S7 Field dependence of the magnetization for $(a) 1$ and $(b) 2$ at 2, 3 and $5 \mathrm{~K}$. dashed line is Brillouin function of $2 \mathrm{Fe}^{\text {III }}$ with $g=2.15$ and $4 \mathrm{Gd}^{\prime \prime \prime}+2 \mathrm{Fe}^{\prime \prime \prime}$ with $g=2.05$, respectively, the solid lines are simulated model using PHI

Fig. S8. Field dependence of the magnetization for $\mathbf{2}$ and $\mathbf{3}$ at 2,3 and $5 \mathrm{~K} \ldots \ldots \ldots \ldots \ldots \ldots . \ldots \ldots$

Fig. S9. Temperature dependencies of the alternating-current susceptibilities for 3 . Lines are

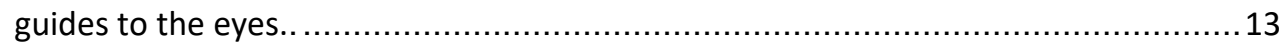

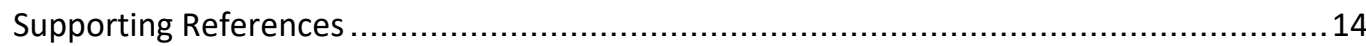


Table S1. Crystallographic data for 1-3.

\begin{tabular}{l|c|c|c}
\hline & $\mathbf{1}$ & $\mathbf{2}$ & $\mathbf{3}$ \\
\hline Formula & $\mathrm{C}_{86} \mathrm{H}_{92} \mathrm{Fe}_{6} \mathrm{~N}_{34} \mathrm{O}_{64} \mathrm{Y}_{4}$ & $\mathrm{C}_{86} \mathrm{H}_{92} \mathrm{Fe}_{6} \mathrm{~N}_{34} \mathrm{O}_{64} \mathrm{Gd}_{4}$ & $2\left\{\mathrm{C}_{86} \mathrm{H}_{92} \mathrm{Fe}_{6} \mathrm{~N}_{34} \mathrm{O}_{64} \mathrm{Dy}_{4}\right.$ \\
$M$ [g mol-1] & $-2 \mathrm{MeCN} \cdot 4 \mathrm{H}_{2} \mathrm{O} \cdot \mathrm{CH}_{2} \mathrm{Cl}_{2}$ & $\cdot 2 \mathrm{MeCN} \cdot 6 \mathrm{H}_{2} \mathrm{O} \cdot \mathrm{CH}_{2} \mathrm{Cl}_{2}$ & $\left.\cdot 2 \mathrm{MeCN} \cdot 10 \mathrm{H}_{2} \mathrm{O} \cdot \mathrm{CH}_{2} \mathrm{Cl}_{2}\right\}$ \\
crystal system & 3555.6310 & 3865.0382 & 7916.1986 \\
space group & monoclinic & monoclinic & monoclinic \\
$a[\AA]$ & $C 2 / c$ & $\mathrm{C} 2 / \mathrm{c}$ & $P 2_{1} / \mathrm{c}$ \\
$b[\AA]$ & $33.0659(15)$ & $33.331(5)$ & $29.254(3)$ \\
$c[\AA][$ & $17.2917(8)$ & $17.375(2)$ & $21.247(2)$ \\
$B\left[^{\circ}\right]$ & $27.457(2)$ & $27.649(4)$ & $24.181(2)$ \\
$V\left[\AA^{3}\right]$ & $123.329(1)$ & $123.408(4)$ & $103.984(3)$ \\
$Z$ & $13117.1(13)$ & $13366(3)$ & $14585(2)$ \\
$\left.\rho(\mathrm{g} \mathrm{cm})^{-3}\right)$ & 4 & 4 & 2 \\
$\mu / \mathrm{mm}^{-1}$ & 1.757 & 1.825 & 1.825 \\
$R_{1}(>2 \sigma)$ & 2.503 & 2.694 & 2.699 \\
$w R_{2}($ all data) & 0.0353 & 0.0354 & 0.0580 \\
$\mathrm{GOF}$ & 0.0773 & 0.0727 & 0.1123 \\
\hline
\end{tabular}

$R_{1}=\sum|| F_{0}|-| F_{c}|| / \sum\left|F_{0}\right| \cdot\left[\sum w\left(F_{o}^{2}-F_{c}^{2}\right)^{2} / w F_{o}^{4}\right]^{1 / 2}$ 
Table S2. Selected distances $(\AA ̊)$ and angles (degree) in the isostructural compounds 1-3.

\begin{tabular}{|c|c|c|c|c|c|c|c|c|}
\hline \multicolumn{2}{|c|}{ Bond dist. } & \multirow{2}{*}{$\frac{\mathrm{Y}(\mathbf{1})}{3.7621(5)}$} & \multirow{2}{*}{$\frac{\mathrm{Gd}(\mathbf{2})}{3.8234(5)}$} & \multicolumn{3}{|c|}{ Bond angles } & \multirow{2}{*}{$\frac{Y(1)}{106.46(8)}$} & \multirow{2}{*}{$\frac{\mathrm{Gd}(\mathbf{2})}{106.71(10)}$} \\
\hline Ln1 & $\operatorname{Ln} 2$ & & & $\operatorname{Ln} 1$ & $\mathrm{O} 1$ & $\operatorname{Ln} 2$ & & \\
\hline $\operatorname{Ln} 1$ & Fe1 & $3.4536(6)$ & $3.4730(8)$ & $\operatorname{Ln} 2^{1}$ & $\mathrm{O} 1$ & $\operatorname{Ln} 1$ & $109.86(9)$ & $109.20(10)$ \\
\hline $\operatorname{Ln} 1$ & $\mathrm{Fe} 2$ & $3.5185(6)$ & $3.5520(5)$ & $\operatorname{Ln} 2^{1}$ & 01 & $\operatorname{Ln} 2$ & $104.77(8)$ & $104.79(10)$ \\
\hline $\operatorname{Ln} 1$ & $\mathrm{Fe} 3$ & $3.2802(6)$ & $3.3147(7)$ & $\operatorname{Ln} 2$ & $\mathrm{O} 2$ & $\operatorname{Ln} 1$ & 102.99(8) & $102.59(10)$ \\
\hline $\operatorname{Ln} 1$ & 01 & $2.332(2)$ & $2.365(3)$ & Fe1 & $\mathrm{O} 5$ & $\operatorname{Ln} 1$ & $97.97(9)$ & $97.86(11)$ \\
\hline $\operatorname{Ln} 1$ & $\mathrm{O} 2$ & $2.408(2)$ & $2.451(3)$ & Fe1 & O5 & $\mathrm{Fe} 2$ & $98.59(9)$ & $98.91(12)$ \\
\hline $\operatorname{Ln} 1$ & $\mathrm{O} 3$ & $2.328(2)$ & $2.371(3)$ & Fe1 & $\mathrm{O} 3$ & $\operatorname{Ln} 1$ & $106.02(10)$ & $105.27(12)$ \\
\hline Ln1 & 05 & $2.516(2)$ & $2.540(3)$ & $\mathrm{Fe} 2$ & $\mathrm{O} 2$ & $\operatorname{Ln} 1$ & $106.31(9)$ & $106.07(11)$ \\
\hline $\operatorname{Ln} 1$ & 07 & $2.248(2)$ & $2.295(3)$ & $\mathrm{Fe} 2$ & $\mathrm{O} 2$ & $\operatorname{Ln} 2$ & $100.82(9)$ & $100.84(11)$ \\
\hline Ln1 & 09 & $2.384(2)$ & $2.423(3)$ & $\mathrm{Fe} 2$ & O5 & $\operatorname{Ln} 1$ & $99.26(8)$ & $99.42(11)$ \\
\hline $\operatorname{Ln} 1$ & $014^{1}$ & $2.343(2)$ & $2.390(3)$ & $\mathrm{Fe} 2$ & 06 & $\operatorname{Ln} 2$ & $104.87(9)$ & $105.33(12)$ \\
\hline $\operatorname{Ln} 1$ & 016 & $2.374(2)$ & $2.410(3)$ & $\mathrm{Fe} 2$ & $\mathrm{O} 4$ & Fe1 & $104.05(10)$ & $104.77(12)$ \\
\hline $\operatorname{Ln} 2$ & $\operatorname{Ln} 2^{1}$ & $3.6675(6)$ & $3.7307(6)$ & $\mathrm{Fe} 3$ & 07 & $\operatorname{Ln} 2$ & $103.05(9)$ & $103.24(11)$ \\
\hline $\operatorname{Ln} 2$ & $\mathrm{Fe} 2$ & $3.3840(6)$ & $3.4264(7)$ & $\mathrm{Fe} 3$ & 08 & $\operatorname{Ln} 1$ & $99.40(9)$ & $99.47(11)$ \\
\hline $\operatorname{Ln} 2$ & $\mathrm{Fe} 3$ & $3.3122(5)$ & $3.3543(7)$ & $\mathrm{Fe} 3$ & $\mathrm{O} 1$ & $\operatorname{Ln} 1$ & $100.86(9)$ & $100.97(11)$ \\
\hline $\operatorname{Ln} 2$ & $\mathrm{O} 1^{1}$ & $2.265(2)$ & $2.308(3)$ & $\mathrm{Fe} 3$ & 01 & $\operatorname{Ln} 2^{1}$ & $101.10(9)$ & $131.64(13)$ \\
\hline $\operatorname{Ln} 2$ & $\mathrm{O} 1$ & $2.364(2)$ & $2.400(3)$ & $\mathrm{Fe} 3$ & $\mathrm{O} 1$ & $\operatorname{Ln} 2$ & $131.56(11)$ & $101.42(11)$ \\
\hline $\operatorname{Ln} 2$ & $\mathrm{O} 2$ & $2.400(2)$ & $2.449(3)$ & & & & & \\
\hline $\operatorname{Ln} 2$ & 06 & $2.298(2)$ & $2.336(3)$ & & & & & \\
\hline $\operatorname{Ln} 2$ & 08 & $2.251(2)$ & $2.292(3)$ & & & & & \\
\hline $\operatorname{Ln} 2$ & 012 & $2.401(2)$ & $2.443(3)$ & & & & & \\
\hline $\operatorname{Ln} 2$ & 013 & $2.476(2)$ & $2.516(3)$ & & & & & \\
\hline $\operatorname{Ln} 2$ & 014 & $2.531(2)$ & $2.571(3)$ & & & & & \\
\hline Fe1 & $\mathrm{O} 3$ & $1.989(2)$ & $1.989(3)$ & & & & & \\
\hline Fe1 & $\mathrm{O} 4$ & $2.013(2)$ & $2.021(3)$ & & & & & \\
\hline Fe1 & 05 & $2.043(2)$ & $2.047(3)$ & & & & & \\
\hline Fe1 & 010 & $2.024(2)$ & $2.030(3)$ & & & & & \\
\hline Fe1 & $\mathrm{N} 1$ & $2.153(3)$ & $2.156(4)$ & & & & & \\
\hline Fe1 & N4 & $1.985(3)$ & $1.988(4)$ & & & & & \\
\hline $\mathrm{Fe} 2$ & $\mathrm{O} 2$ & $1.977(2)$ & $1.980(3)$ & & & & & \\
\hline $\mathrm{Fe} 2$ & $\mathrm{O} 4$ & $1.959(2)$ & $1.959(3)$ & & & & & \\
\hline $\mathrm{Fe} 2$ & 05 & $2.088(2)$ & $2.102(3)$ & & & & & \\
\hline $\mathrm{Fe} 2$ & 06 & $1.963(2)$ & $1.964(3)$ & & & & & \\
\hline $\mathrm{Fe} 2$ & 011 & $1.980(2)$ & $1.988(3)$ & & & & & \\
\hline $\mathrm{Fe} 2$ & N2 & $2.155(3)$ & $2.166(4)$ & & & & & \\
\hline $\mathrm{Fe} 3$ & 01 & $1.909(2)$ & $1.916(3)$ & & & & & \\
\hline $\mathrm{Fe} 3$ & 07 & $2.049(2)$ & $1.980(3)$ & & & & & \\
\hline $\mathrm{Fe} 3$ & 08 & $1.973(2)$ & $2.044(3)$ & & & & & \\
\hline $\mathrm{Fe} 3$ & 015 & $2.056(2)$ & $2.066(3)$ & & & & & \\
\hline $\mathrm{Fe} 3$ & N3 & $2.205(3)$ & $2.213(4)$ & & & & & \\
\hline $\mathrm{Fe} 3$ & N7 & $2.066(3)$ & $2.075(4)$ & & & & & \\
\hline Fe1 & $\mathrm{Fe} 2$ & $3.132(1)$ & $3.132(1)$ & & & & & \\
\hline
\end{tabular}

$1_{1 / 2-X, 1 / 2-Y,-Z}$ 


\begin{tabular}{|c|c|c|c|c|c|c|c|c|c|}
\hline & & Distances & & & Distances & & & & Angles \\
\hline Dy1A & Dy2A & $3.8015(6)$ & Dy1B & Dy2B & $3.8019(6)$ & Dy1B & O1B & Dy2B $^{2}$ & $105.35(17)$ \\
\hline Dy1A & $D^{2} 2 A^{1}$ & $3.7747(7)$ & Dy1B & Dy2B ${ }^{2}$ & $3.7582(6)$ & Dy1B & O14B & Dy2B & $101.57(18)$ \\
\hline Dy1A & $\mathrm{Fe} 2 \mathrm{~A}$ & $3.4835(14)$ & Dy1B & $\mathrm{Fe} 2 \mathrm{~B}^{2}$ & $3.4910(1)$ & Dy2B & O1B & Dy2B ${ }^{2}$ & $105.20(19)$ \\
\hline Dy1A & $\mathrm{Fe} 3 \mathrm{~A}$ & $3.3052(13)$ & Dy1B & $\mathrm{Fe} 3 \mathrm{~B}$ & $3.3056(11)$ & Dy1B ${ }^{2}$ & O2B & Dy2B & $101.89(17)$ \\
\hline Dy1A & O1A & $2.353(6)$ & Dy1B & O1B & $2.363(5)$ & Fe1B & O5B & $\mathrm{Fe} 2 \mathrm{~B}$ & $99.6(2)$ \\
\hline Dy1A & $\mathrm{O} 2 \mathrm{~A}$ & $2.409(6)$ & Dy1B & $\mathrm{O} 2 \mathrm{~B}^{2}$ & $2.400(5)$ & Fe1B & O5B & Dy1B ${ }^{2}$ & $98.7(2)$ \\
\hline Dy1A & O3A & $2.343(5)$ & Dy1B & $\mathrm{O}^{3} \mathrm{~B}^{2}$ & $2.329(5)$ & Fe1B & O3B & Dy1B 2 & $106.9(2)$ \\
\hline Dy1A & O5A & $2.516(6)$ & Dy1B & $05 \mathrm{~B}^{2}$ & $2.517(5)$ & $\mathrm{Fe} 2 \mathrm{~B}$ & $\mathrm{O} 2 \mathrm{~B}$ & Dy1B ${ }^{2}$ & $105.2(2)$ \\
\hline Dy1A & O7A & $2.273(5)$ & Dy1B & O7B & $2.260(5)$ & $\mathrm{Fe} 2 \mathrm{~B}$ & $\mathrm{O} 2 \mathrm{~B}$ & Dy2B & $100.82(19)$ \\
\hline Dy1A & O9A & $2.358(6)$ & Dy1B & O9B & $2.361(5)$ & $\mathrm{Fe} 2 \mathrm{~B}$ & O5B & Dy1B 2 & $98.7(2)$ \\
\hline Dy1A & $014 A^{1}$ & $2.350(5)$ & Dy1B & O14B & $2.350(6)$ & $\mathrm{Fe} 2 \mathrm{~B}$ & O6B & Dy2B & $105.9(2)$ \\
\hline Dy1A & O16A & $2.344(6)$ & Dy1B & O16B & $2.366(5)$ & $\mathrm{Fe} 2 \mathrm{~B}$ & O4B & Fe1B & $105.3(2)$ \\
\hline Dy2A & $\mathrm{Dy}_{2 \mathrm{~A}^{1}}$ & $3.6346(8)$ & Dy2B & Dy2B ${ }^{2}$ & $3.6730(7)$ & $\mathrm{Fe} 3 \mathrm{~B}$ & O7B & Dy $2 B^{2}$ & $102.1(2)$ \\
\hline Dy2A & $\mathrm{Fe} 2 \mathrm{~A}^{1}$ & $3.4220(13)$ & Dy2B & $\mathrm{Fe} 2 \mathrm{~B}$ & $3.4202(11)$ & $\mathrm{Fe} 3 \mathrm{~B}$ & O8B & Dy1B & $100.5(2)$ \\
\hline Dy2A & $\mathrm{Fe} 3 \mathrm{~A}^{1}$ & $3.3073(13)$ & Dy2B & $\mathrm{Fe}^{3} \mathrm{~B}^{2}$ & $3.3093(12)$ & $\mathrm{Fe} 3 \mathrm{~B}$ & O1B & Dy1B & $100.8(2)$ \\
\hline Dy2A & $01 A^{1}$ & $2.363(6)$ & Dy2B & O1B & $2.260(5)$ & $\mathrm{Fe} 3 \mathrm{~B}$ & O1B & Dy2B & $131.3(2)$ \\
\hline Dy2A & O1A & $2.259(5)$ & Dy2B & $01 B^{2}$ & $2.363(5)$ & $\mathrm{Fe} 3 \mathrm{~B}$ & O1B & Dy2B ${ }^{2}$ & $101.0(2)$ \\
\hline Dy2A & $\mathrm{O} 2 \mathrm{~A}^{1}$ & $2.447(6)$ & Dy2B & $\mathrm{O} 2 \mathrm{~B}$ & $2.440(5)$ & Dy1A & $01 \mathrm{~A}$ & Dy $2 A^{1}$ & $106.3(2)$ \\
\hline Dy2A & $06 A^{1}$ & $2.291(5)$ & Dy2B & O6B & $2.307(6)$ & Dy1A & $\mathrm{O} 2 \mathrm{~A}$ & Dy $2 A^{1}$ & $102.0(2)$ \\
\hline Dy2A & $08 \mathrm{~A}^{1}$ & $2.259(5)$ & Dy2B & $08 \mathrm{~B}^{2}$ & $2.289(5)$ & Dy2A & O1A & Dy1A & $109.9(2)$ \\
\hline Dy2A & $\mathrm{O} 12 \mathrm{~A}$ & $2.385(6)$ & Dy2B & O12B & $2.451(5)$ & Dy $2 A$ & $01 \mathrm{~A}$ & Dy $2 A^{1}$ & $103.7(2)$ \\
\hline Dy2A & O13A & $2.503(6)$ & Dy2B & O13B & $2.450(5)$ & Dy1A & 014A & Dy2A & $101.9(2)$ \\
\hline Dy2A & O14A & $2.510(6)$ & Dy2B & O14B & $2.554(5)$ & Fe1A & $05 \mathrm{~A}$ & $\mathrm{Fe} 2 \mathrm{~A}$ & $99.2(2)$ \\
\hline Fe1A & O3A & $1.973(5)$ & $\mathrm{Fe} 1 \mathrm{~B}$ & О3В & $2.005(5)$ & Fe1A & O5A & Dy1A & $98.4(2)$ \\
\hline Fe1A & O4A & $2.011(6)$ & $\mathrm{Fe} 1 \mathrm{~B}$ & O8B & $2.011(5)$ & Fe1A & O3A & Dy1A & $107.3(2)$ \\
\hline Fe1A & 05A & $2.066(6)$ & Fe1B & O5B & $2.062(5)$ & $\mathrm{Fe} 2 \mathrm{~A}$ & $\mathrm{O} 2 \mathrm{~A}$ & Dy1A & $104.9(3)$ \\
\hline Fe1A & O10A & $2.046(6)$ & Fe1B & O10B & $2.026(6)$ & $\mathrm{Fe} 2 \mathrm{~A}$ & $\mathrm{O} 2 \mathrm{~A}$ & Dy $2 A^{1}$ & $101.0(2)$ \\
\hline $\mathrm{Fe} 1 \mathrm{~A}$ & $\mathrm{~N} 1 \mathrm{~A}$ & $2.152(7)$ & $\mathrm{Fe} 1 \mathrm{~B}$ & N1B & $2.162(7)$ & $\mathrm{Fe} 2 \mathrm{~A}$ & O5A & Dy1A & $98.1(2)$ \\
\hline Fe1A & $\mathrm{N} 4 \mathrm{~A}$ & $1.997(8)$ & $\mathrm{Fe} 1 \mathrm{~B}$ & N4B & $1.994(8)$ & $\mathrm{Fe} 2 \mathrm{~A}$ & O6A & Dy $2 A^{1}$ & $106.7(2)$ \\
\hline $\mathrm{Fe} 2 \mathrm{~A}$ & $\mathrm{O} 2 \mathrm{~A}$ & $1.972(5)$ & $\mathrm{Fe} 2 \mathrm{~B}$ & O2B & $1.982(5)$ & $\mathrm{Fe} 2 \mathrm{~A}$ & $\mathrm{O} 4 \mathrm{~A}$ & Fe1A & $105.8(3)$ \\
\hline $\mathrm{Fe} 2 \mathrm{~A}$ & O4A & $1.951(6)$ & $\mathrm{Fe} 2 \mathrm{~B}$ & O4B & $1.954(5)$ & $\mathrm{Fe} 3 \mathrm{~A}$ & O7A & Dy $2 A^{1}$ & $102.5(2)$ \\
\hline $\mathrm{Fe} 2 \mathrm{~A}$ & O5A & $2.082(7)$ & $\mathrm{Fe} 2 \mathrm{~B}$ & O6B & $1.971(5)$ & $\mathrm{Fe} 3 \mathrm{~A}$ & O8A & Dy1A & $99.8(2)$ \\
\hline $\mathrm{Fe} 2 \mathrm{~A}$ & O6A & $1.967(5)$ & $\mathrm{Fe} 2 \mathrm{~B}$ & O5B & $2.066(5)$ & $\mathrm{Fe} 3 \mathrm{~A}$ & $01 \mathrm{~A}$ & Dy1A & $101.1(2)$ \\
\hline $\mathrm{Fe} 2 \mathrm{~A}$ & O11A & $1.986(7)$ & $\mathrm{Fe} 2 \mathrm{~B}$ & O11B & $1.971(6)$ & $\mathrm{Fe} 3 \mathrm{~A}$ & $01 \mathrm{~A}$ & Dy2A & $132.6(3)$ \\
\hline $\mathrm{Fe} 2 \mathrm{~A}$ & $\mathrm{~N} 2 \mathrm{~A}$ & $2.189(9)$ & $\mathrm{Fe} 2 \mathrm{~B}$ & $\mathrm{~N} 2 \mathrm{~B}$ & $2.170(7)$ & $\mathrm{Fe} 3 \mathrm{~A}$ & $01 \mathrm{~A}$ & Dy $2 A^{1}$ & $100.8(2)$ \\
\hline $\mathrm{Fe} 3 \mathrm{~A}$ & O1A & $1.913(5)$ & $\mathrm{Fe} 3 \mathrm{~B}$ & O1B & $1.910(5)$ & & & & \\
\hline $\mathrm{Fe} 3 \mathrm{~A}$ & O7A & $2.044(6)$ & $\mathrm{Fe} 3 \mathrm{~B}$ & O4B & $2.035(6)$ & & & & \\
\hline $\mathrm{Fe} 3 \mathrm{~A}$ & O8A & $1.976(6)$ & $\mathrm{Fe} 3 \mathrm{~B}$ & O7B & $1.958(6)$ & & & & \\
\hline $\mathrm{Fe} 3 \mathrm{~A}$ & O15A & $2.074(6)$ & $\mathrm{Fe} 3 \mathrm{~B}$ & O15B & $2.062(6)$ & & & & \\
\hline $\mathrm{Fe} 3 \mathrm{~A}$ & N3A & $2.208(7)$ & $\mathrm{Fe} 3 \mathrm{~B}$ & N3B & $2.199(7)$ & & & & \\
\hline $\mathrm{Fe} 3 \mathrm{~A}$ & N7A & $2.074(7)$ & $\mathrm{Fe} 3 \mathrm{~B}$ & N7B & $2.056(6)$ & & & & \\
\hline
\end{tabular}


Table S3. Shape measurements for $\operatorname{Ln}($ III) coordination environments in compounds 1-3.

\begin{tabular}{l|c|c|c|c|c} 
& SAPR-8 & TDD-8 & JBTPR-8 & BTPR-8 & JSD-8 \\
\hline $\mathrm{Y}_{1}$ & 0.839 & 1.464 & 2.368 & 2.048 & 3.969 \\
$\mathrm{Y}_{2}$ & 3.749 & 2.290 & 4.266 & 3.51 & 5.74 \\
$\mathrm{Gd}_{1}$ & 0.869 & 1.429 & 2.481 & 2.11 & 3.979 \\
$\mathrm{Gd}_{2}$ & 3.804 & 2.398 & 4.341 & 3.596 & 5.803 \\
$\mathrm{Dy}_{1 \mathrm{~A}}$ & 0.974 & 1.579 & 2.128 & 1.795 & 4.177 \\
$\mathrm{Dy}_{1 \mathrm{~B}}$ & 0.647 & 1.910 & 2.066 & 1.766 & 4.332 \\
$\mathrm{Dy}_{2 \mathrm{~A}}$ & 4.340 & 2.309 & 3.684 & 3.089 & 5.526 \\
$\mathrm{Dy}_{2 \mathrm{~B}}$ & 3.658 & 2.323 & 4.419 & 3.624 & 5.805
\end{tabular}

SAPR-8: square antiprism $\left(D_{4 d}\right)$; TDD-8: triangular dodecahedron $\left(D_{2 d}\right)$; JBTPR-8: Biaugmented trigonal prism J50 $\left(C_{2 v}\right)$; BTPR-8: biaugmented trigonal prism $\left(C_{2 v}\right)$; JSD-8: Snub diphenoid J84 $\left(D_{2 d}\right)$. The least distorted geometries have been highlighted in yellow. All these measurements were carried out by SHAPE. ${ }^{1}$ 

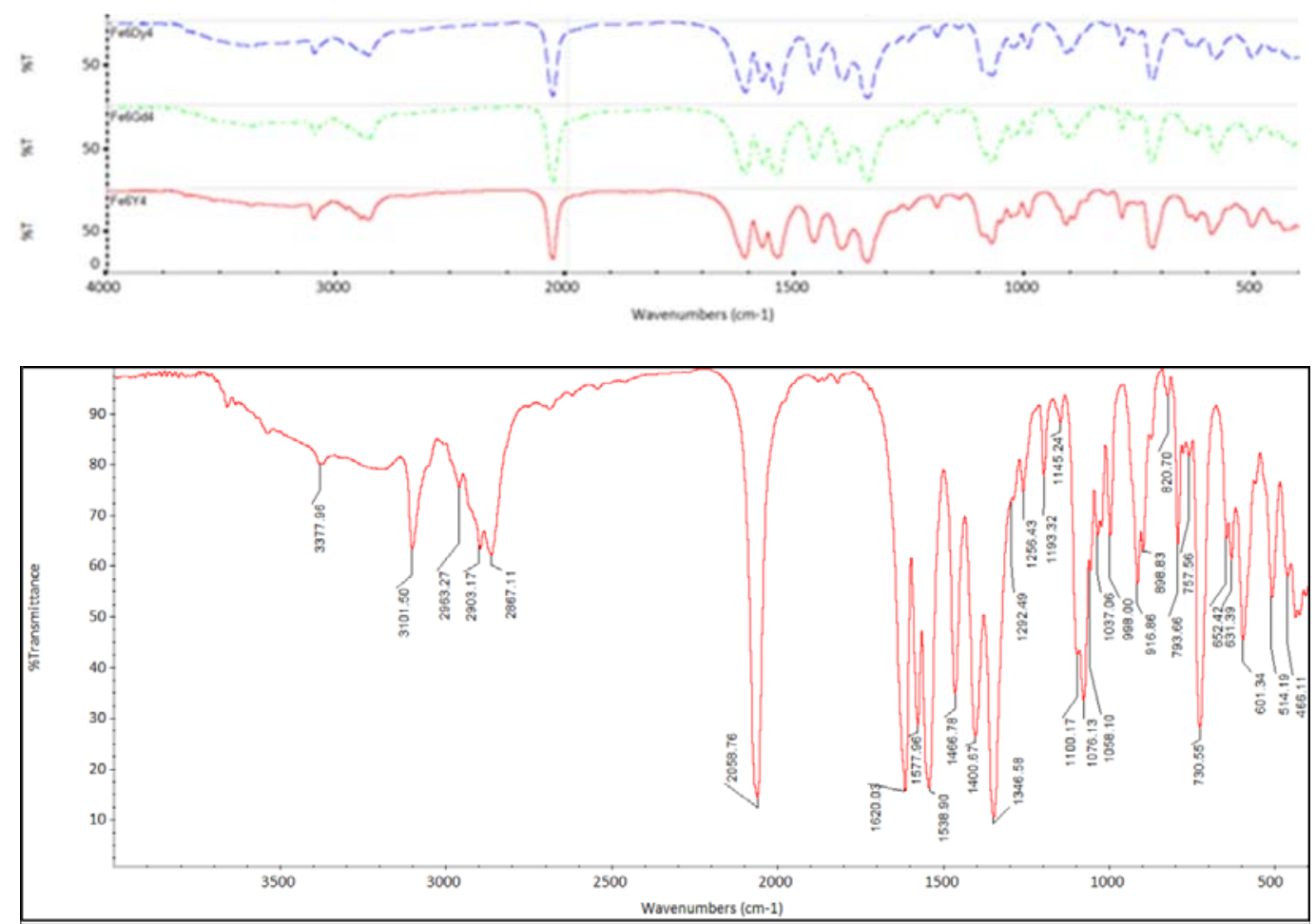

Fig. S1. Comparative representation of FTIR spectra of 1-3 (above), important peaks in 1 (below).

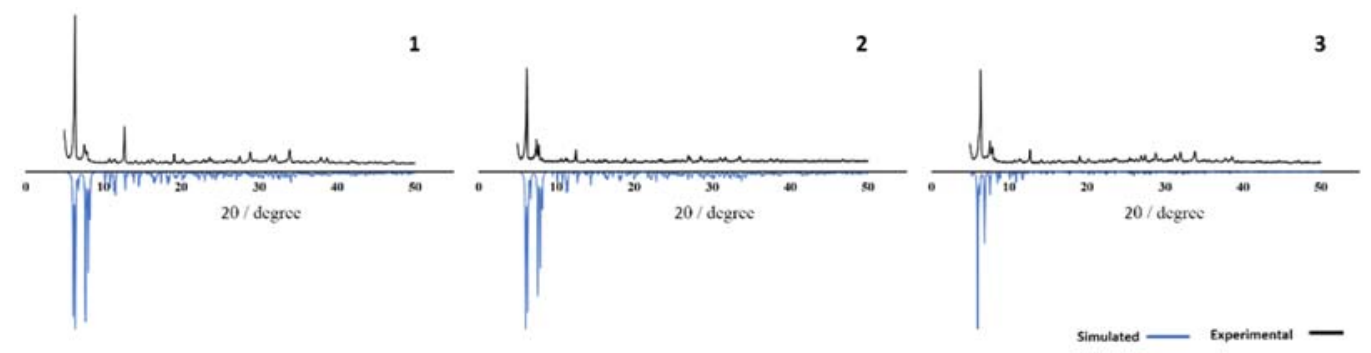

Fig. S2. Experimental and simulated powder X-ray diffraction (PXRD) patterns for 1-3. 

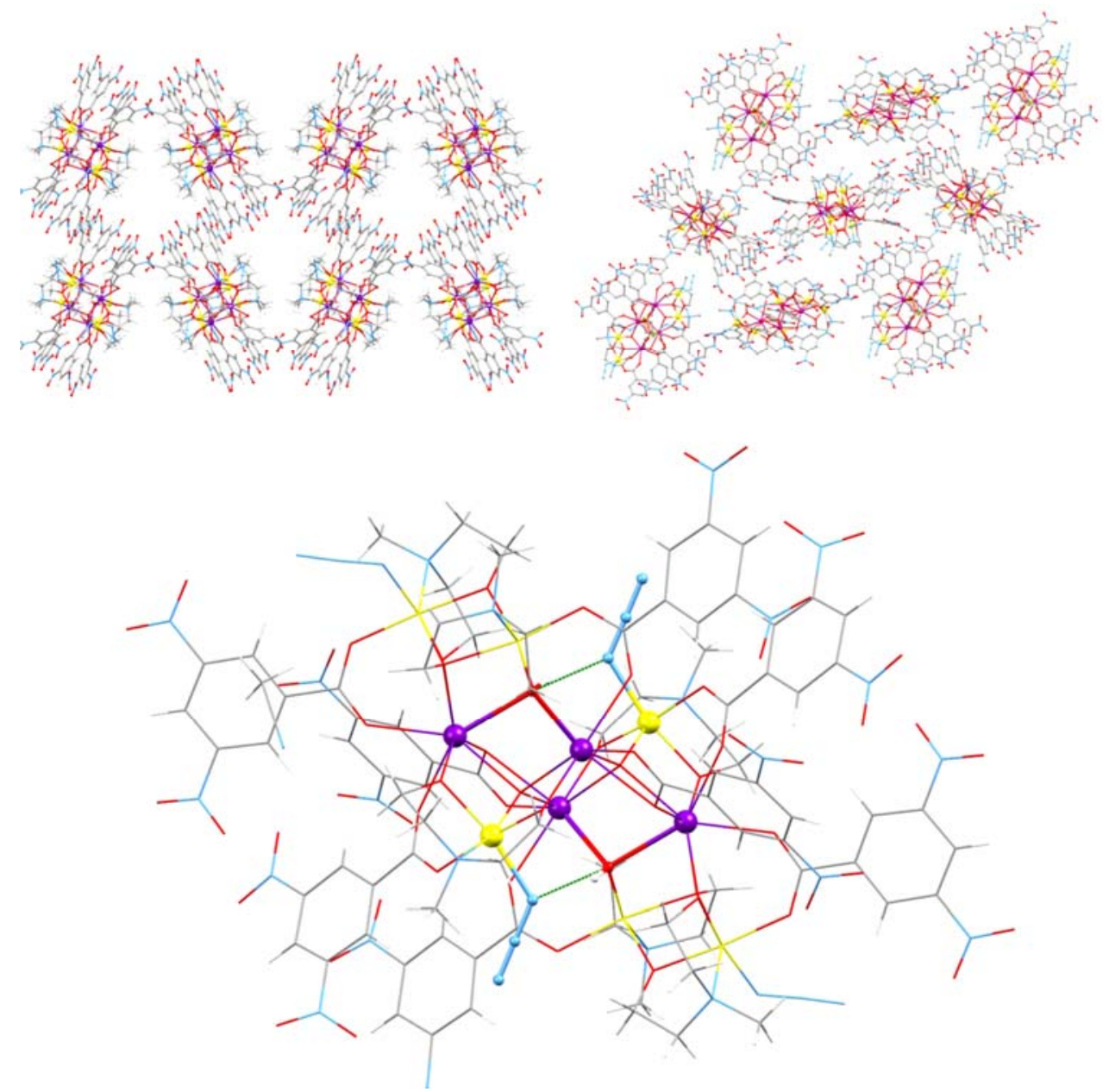

Fig. S3. (Above) views of 1 along the plane ca. $(-1,0,1)$ and 3 along the plane ca. $(-1,1.5,-1)$, showing a packing pattern and the solvent accessible voids; iron: yellow, nitrogen: blue, oxygen: red, Y/Dy : pink and violet. Below) A molecular unit of 1, showing an intramolecular interaction (green dashed line) between the azide ligand and hydroxyl hydrogen. 

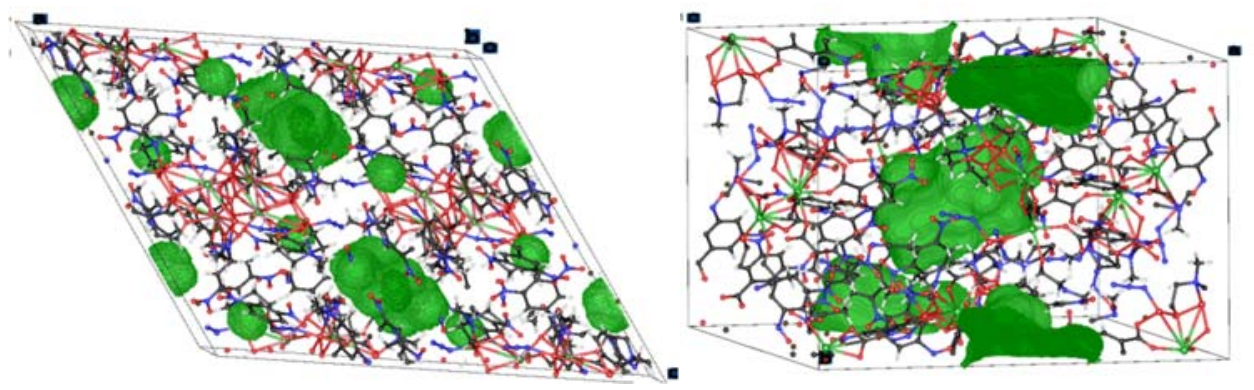

Fig S4. Perspective views of 2 and 3 showing the available internal voids obtained by Olex2. ${ }^{2}$ 

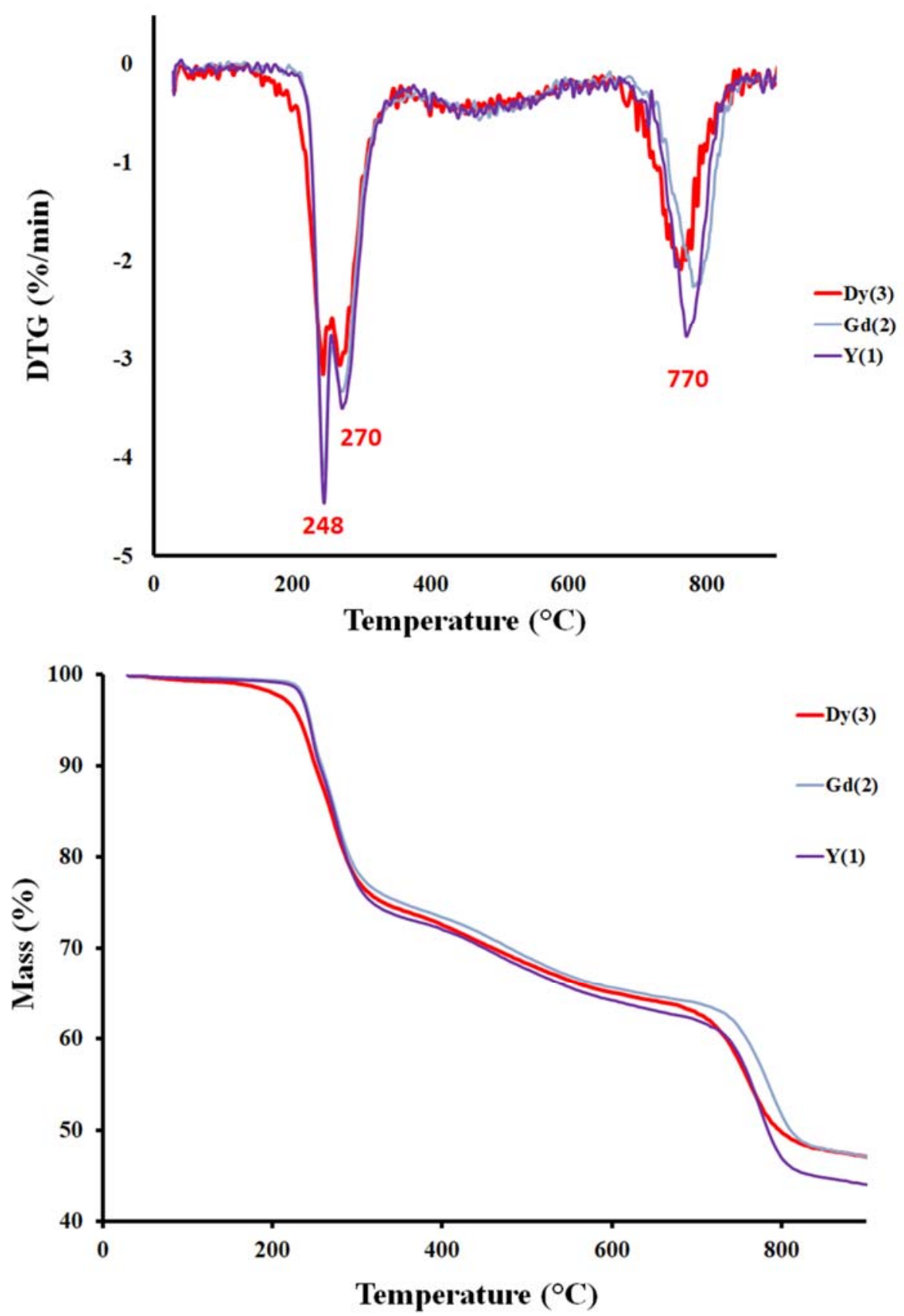

Fig. S5. TGA and DTG curves for 1-3. 


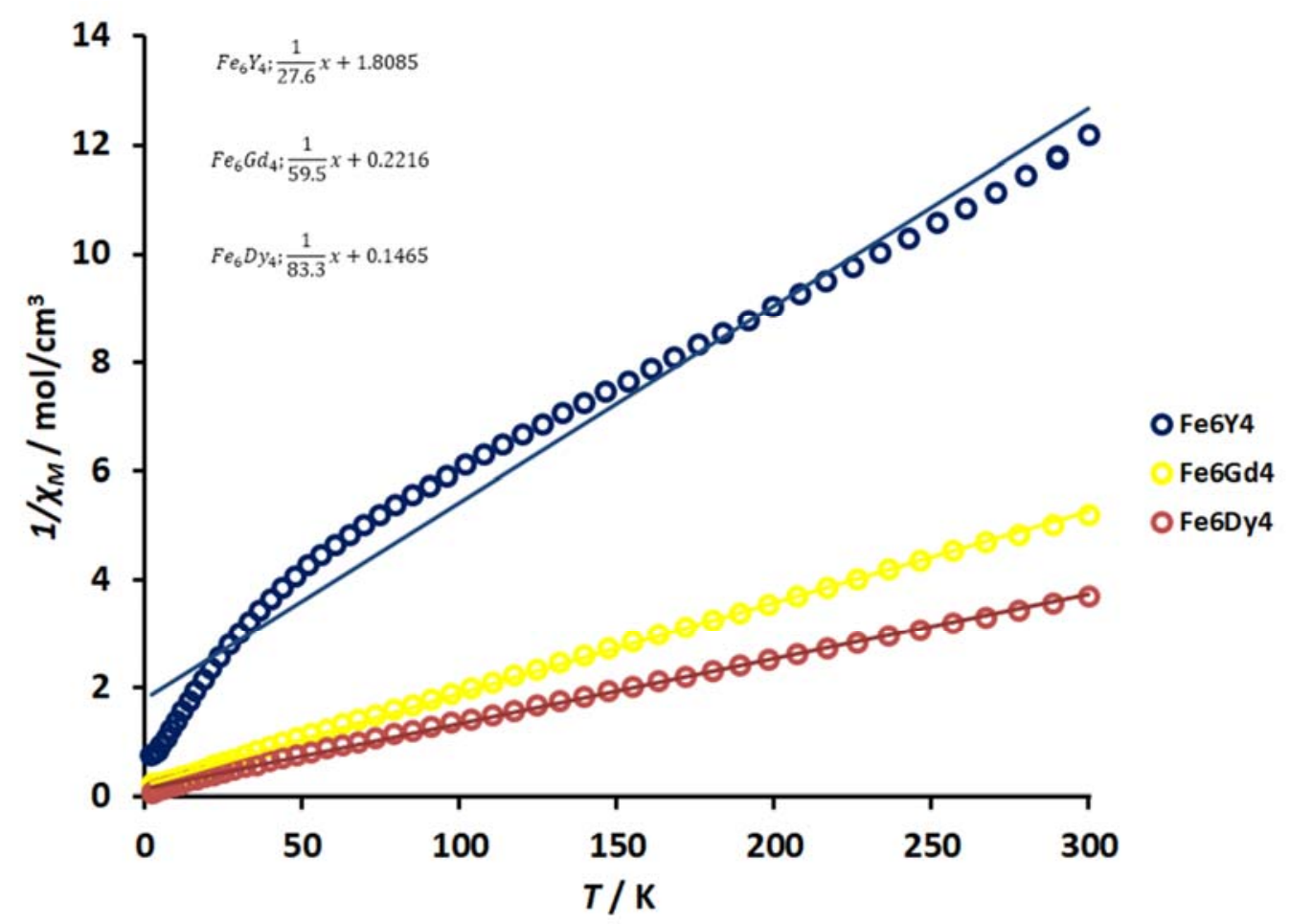

Fig. S6. $\chi_{M}{ }^{-1}$ versus $T$ plots data for $1-3$; inset shows the trendlines equations. 

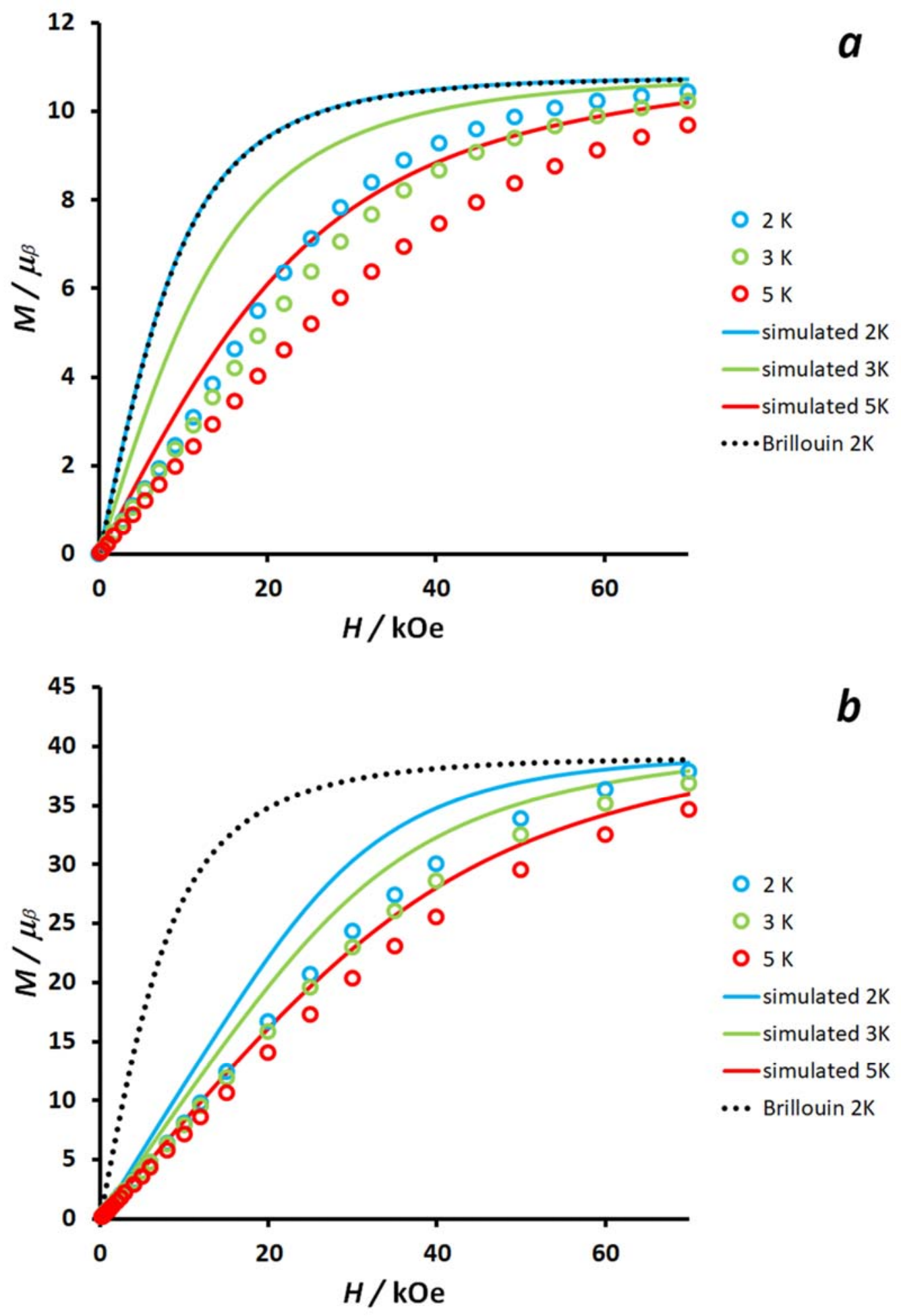

Fig. S7. Field dependence of the magnetization for (a) 1 and (b) 2 at 2, 3 and $5 \mathrm{~K}$. dashed line is Brillouin function of $2 \mathrm{Fe}^{\mathrm{III}}$ with $g=2.15$ and $4 \mathrm{Gd}^{\mathrm{III}}+2 \mathrm{Fe}^{\mathrm{III}}$ with $g=$ 2.05, respectively, the solid lines are simulated model using $\mathrm{PHI}^{3}$. 


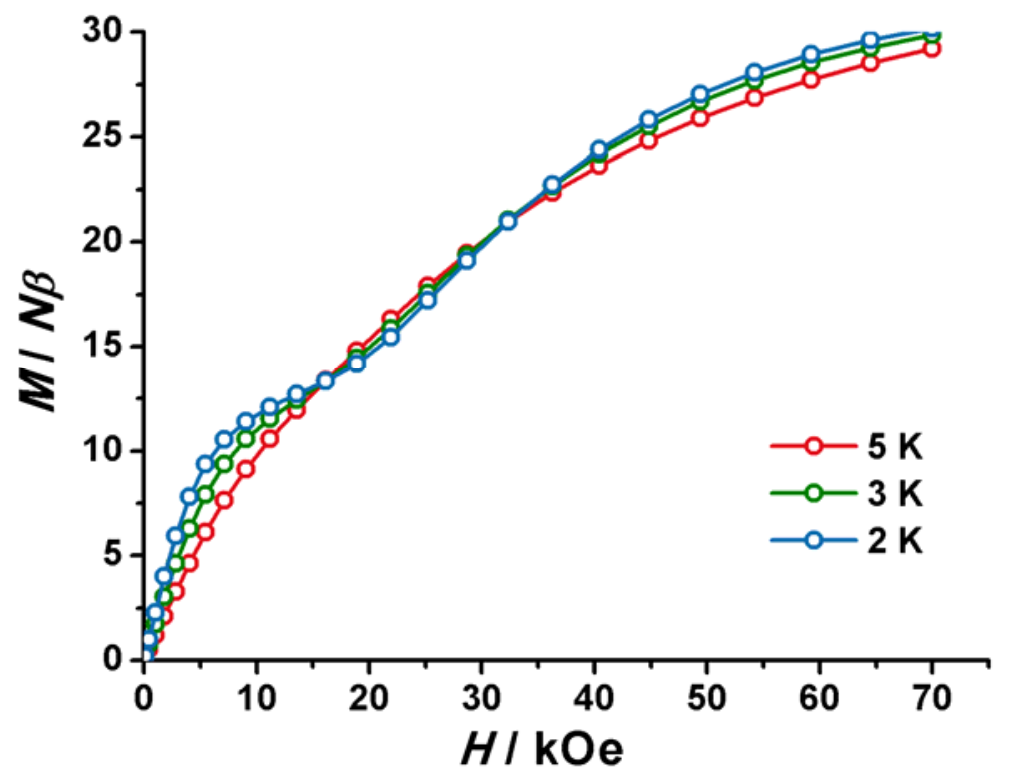

Fig. S8. Field dependence of the magnetization for 3 at 2, 3 and $5 \mathrm{~K}$. 


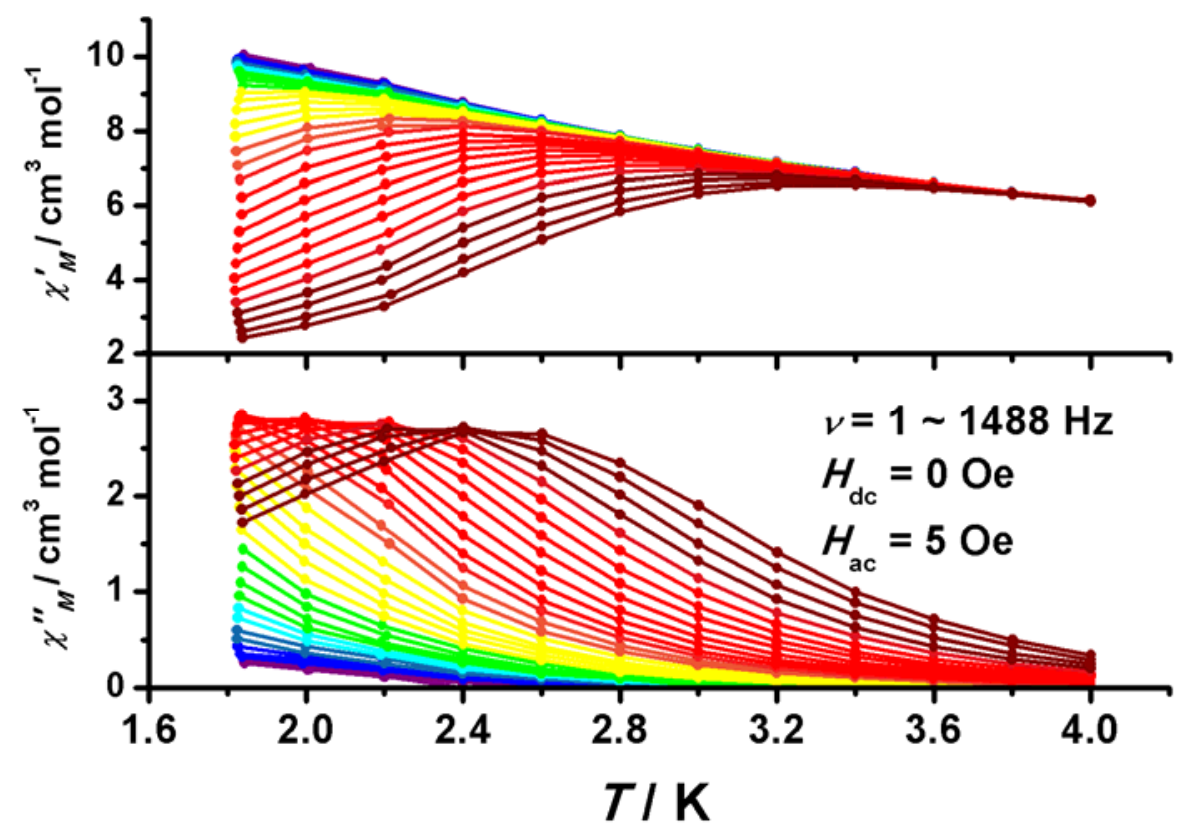

Fig. S9. Temperature dependencies of the alternating-current susceptibilities for 3. Lines are guides to the eyes.

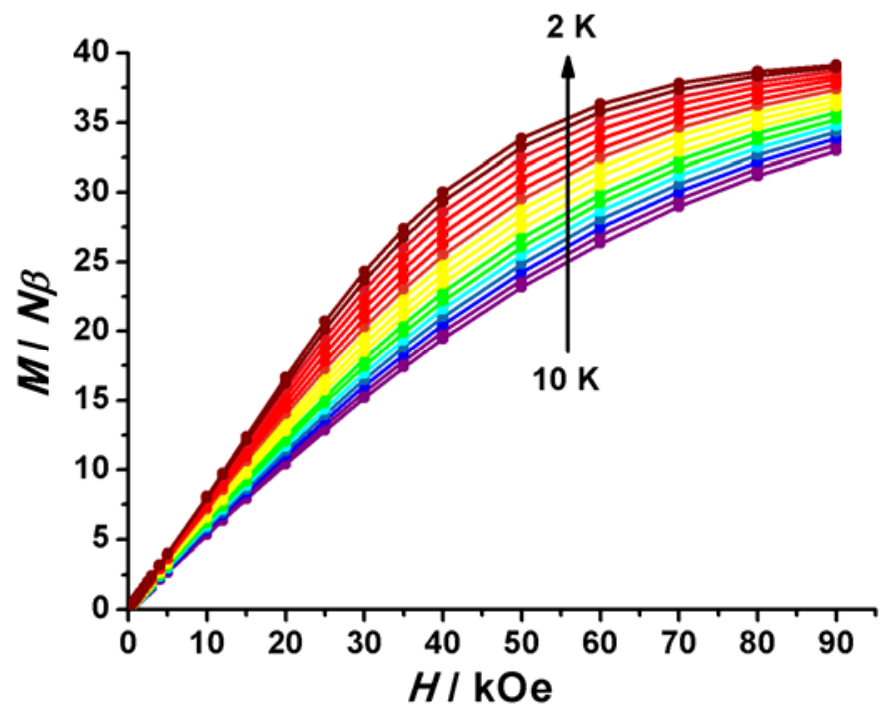

Fig. S10. The magnetization $(M)$ of 2 at different fields and temperatures. 


\section{Supporting References}

1. M. Llunell, D. Casanova, J. Cirera, J. M. Bofill, P. Alemany, S. Alvarez, M. Pinsky and D. Avnir, SHAPE 2.1, University of Barcelona, Barcelona, 2013.

2. O. V. Dolomanov, L. J. Bourhis, R. J. Gildea, J. A. K. Howard and H. Puschmann, J. Appl. Crystallogr., 2009, 42, 339-341.

3. N. F. Chilton, R. P. Anderson, L. D. Turner, A. Soncini and K. S. Murray, J. Comput. Chem., 2013, 34, 1164-1175. 